\title{
Género y la formalización de las comunidades nativas en la Amazonía peruana
}

\author{
Anne M Larson, lliana Monterroso y Pamela Cantuarias
}

\section{Mensajes clave}

- Las mujeres indígenas son afectadas no solo por la seguridad de tenencia de su tierra colectiva sino también por su condición de mujeres. Por ello, tanto las leyes nacionales como las normas comunales son de suma importancia.

- La legislación peruana protege a las mujeres y promueve la equidad en términos generales, pero no en las legislaciones específicas sobre tenencia de tierras o referida a comunidades nativas.

- Las entrevistas con funcionarios de gobierno a cargo de la formalización de la tierra en el Perú revelan un menor conocimiento de temas referidos al género en relación a otros funcionarios similares en Uganda, Indonesia y Nepal.

- Los resultados de la encuesta de hogares muestran diferencias de género importantes, en el uso y el manejo forestal, así como en la toma de decisiones. También en la percepción sobre la equidad de las normas, la seguridad de tenencia y los factores de inseguridad relacionados a los procesos de titulación.

- Para poder avanzar, es necesario promover el fortalecimiento de capacidades de las mujeres para que participen más en los procesos de titulación, así como una mayor sensibilización sobre género para que se incorporen las perspectivas de las mujeres. Además, capacitación y reflexión sobre género para el gobierno, las federaciones indígenas y las comunidades; al igual que una mayor articulación entre funcionarios gubernamentales y comunidades, con el respaldo de las ONG y las organizaciones y federaciones de mujeres.

\section{Introducción}

Se reconoce ampliamente que los derechos de las mujeres a la tierra y a los bosques no han sido protegidos suficientemente por los gobiernos nacionales. El Objetivo 5 de los Objetivos de Desarrollo Sostenible (ODS) menciona específicamente el acceso de las mujeres a la propiedad y control sobre la tierra como uno de los factores para asegurar igualdad de oportunidades y su participación plena y efectiva. Con frecuencia, las mujeres indígenas son 'doblemente marginadas' debido a su etnicidad y género (Keene y Ginsburg 2017). Sus derechos a las tierras colectivas afrontan una mayor complejidad ya que como mujeres indígenas están sujetas tanto a las leyes nacionales como a las costumbres y normas locales.

\section{El Perú ha reconocido los derechos colectivos de} comunidades indígenas a la tierra y al bosque en la Amazonía por más de cuatro décadas. Más de 1300 comunidades nativas han sido tituladas, y aún quedan unas 600 por titular (SICNA-IBC 2016), además casi una docena de proyectos de titulación se encuentran en proceso actualmente (Monterroso y Larson 2018). Este Infobrief hace un balance de los derechos colectivos de las mujeres a la tierra en Perú, como parte del Estudio Comparativo Global en Reformas de Tenencia en Tierras Forestales de CIFOR. Este proyecto combinó múltiples métodos de investigación por un período de 5 años para entender la historia, el contexto, la implementación y los resultados de las reformas de tenencia en las comunidades nativas peruanas, enfocándose especialmente en las regiones de Loreto y Madre de Dios. Este documento resume los hallazgos relativos al género en estos diversos ámbitos.

\section{Recolección y análisis de la información}

Este informe utiliza los diferentes métodos aplicados en el estudio para compilar y sintetizar algunos de los datos claves y relevantes sobre género que se obtuvieron entre el 2014 y 2017. Esto incluye: (1) una encuesta a funcionarios de gobierno a cargo de la implementación (32 personas identificadas como actores clave en las etapas de implementación, ver Monterroso et al. 2018 y Notess et al. 2017), así como un conjunto de entrevistas complementarias con organizaciones no gubernamentales (ONG) y otros 
actores involucrados (23 personas, datos no publicados). (2) Información de campo e informes que analizan entrevistas con 43 informantes clave y 44 grupos focales -la mitad con mujeres y la otra mitad con hombres- en 22 comunidades estudiadas en Madre de Dios y Loreto, de las cuales 18 han sido tituladas. (3) Encuestas de hogares con igual número de hombres y mujeres (1006 participantes en total) de estas 22 comunidades. (4) Talleres de Análisis de Prospectiva Participativa (APP) para la elaboración de escenarios con diversos actores (Bourgeois et al. 2017), pero en este informe se pone énfasis en un taller realizado con 38 mujeres representantes de diferentes asociaciones indígenas de todo el país (Zamora y Monterroso 2017). También se consideran algunos recursos secundarios, una revisión legal y otras publicaciones del proyecto.

Este informe está organizado por temas, más que por métodos. Empieza con el análisis de cómo se considera el género en las leyes nacionales y luego examina cómo este se aborda desde la perspectiva de los implementadores del gobierno (y algunos no gubernamentales) de los procesos de titulación en Perú. Las siguientes secciones hacen uso de los resultados a nivel de comunidad para debatir sobre género en relación a la membresía a la comunidad, las mujeres en los bosques y las mujeres en la toma de decisiones. El siguiente apartado da una mirada a las percepciones en la seguridad de tenencia desde las diferencias de género, con base en el taller de APP y las encuestas en las comunidades. El informe se centra principalmente en Perú, pero menciona algunos puntos de comparación con los otros países estudiados cuando resulta de ayuda para profundizar el análisis.

\section{Consideraciones de género en la formalización colectiva según la legislación peruana}

Las leyes peruanas han adoptado instrumentos internacionales e incorporado consideraciones de justicia y equidad de género como principios en la normativa nacional. Perú firmó la Convención sobre la eliminación de todas las formas de discriminación contra la mujer (CEDAW, por sus siglas en inglés) y adoptó las Directrices voluntarias sobre la gobernanza responsable de la tenencia de la tierra (VGGT, por sus siglas en inglés), que establecen que las mujeres y las niñas deben contar con derechos equitativos de tenencia y acceso a la tierra y a los bosques, independientemente de su estado civil y marital. Perú también ha ratificado la Agenda 2030 sobre los Objetivos de Desarrollo Sostenible, que busca empoderar a los pueblos indígenas y asegurar los derechos de tenencia de la tierra de las mujeres.

A nivel nacional, la Constitución Nacional garantiza la misma protección y algunas leyes relevantes, como la Ley Forestal y de Fauna Silvestre y la Ley Orgánica de Gobiernos Regionales, establecen la igualdad e inclusión social como principios importantes. Al igual que varios otros países latinoamericanos, el Perú reconoce formalmente derechos igualitarios de herencia para las mujeres (hijas, viudas y mujeres en uniones consensuales acreditadas) (Keene y Ginsburg 2017). Sin embargo, la legislación sobre tenencia de tierras no tiene ninguna provisión específica relacionada a género (Soria 2017). La membresía de las mujeres se garantiza en leyes específicas sobre las comunidades campesinas, pero no existe lo mismo para las comunidades nativas (Keene y Ginsburg 2017).

\section{Género en la implementación de la titulación para las comunidades nativas}

Luego de un limitado avance por más de una década, el Gobierno peruano ha reiniciado, recientemente, la titulación de las comunidades nativas (Monterroso et al. 2017). Para 2014, un conjunto de proyectos, especialmente aquellos respaldados por iniciativas sobre cambio climático, habían empezado a financiar la titulación (Monterroso y Larson 2018).

Este análisis se basa en la encuesta, anteriormente mencionada, aplicada a funcionarios de gobierno a nivel nacional y subnacional y de organizaciones no gubernamentales, así como de otras entidades involucradas en algunas de estas iniciativas.

A pesar de que la muestra no es aleatoria, la encuesta a los funcionarios gubernamentales nacionales y subnacionales a cargo de la titulación sugiere que el proceso se encuentra dominada por los hombres: $72 \%$ de los entrevistados fueron hombres, en comparación con el $28 \%$ de mujeres. Cuando se les pidió establecer los objetivos principales de las reformas (más de una respuesta era posible), solo uno de los implementadores gubernamentales (3\% de todos los funcionarios de gobierno) señaló que los principales objetivos de las reformas incluían el reconocimiento y fortalecimiento de los derechos de los grupos vulnerables, como mujeres y pueblos indígenas. En comparación, esto resulta mucho menor que los resultados obtenidos en Nepal (38\%) y Uganda (23\%), pero similar a las respuestas obtenidas en Indonesia (donde solo 4\% de los encuestados consideró esta opción). Entre los entrevistados de las ONG en Perú, cinco mencionaron este objetivo.

Con relación a las actividades de apoyo al acceso a los bosques, solo $14 \%$ de los funcionarios públicos involucrados en tales actividades (3 de 21) dijo que brindaban consideración especial a las mujeres. Cuando se les preguntó sobre las metas especiales de la reforma, solo 9\% (3 de 32) de los implementadores consideró a las mujeres. Se obtuvieron resultados similares en Indonesia (7\%), sin embargo, estos fueron mejores en Uganda (90\%) y Nepal (62\%). Únicamente un funcionario de gobierno mencionó la exclusión de las mujeres y otros grupos vulnerables como parte de los cuatro principales obstáculos para implementar la titulación (un encuestado de ONG también señaló esto). Ningún oficial gubernamental reportó participar en la incorporación de la perspectiva de género, en comparación con cuatro entrevistados de las ONG.

Cuando se preguntó sobre las necesidades o demandas específicas de las comunidades en las que trabajan, la debilidad en los derechos de las mujeres o la exclusión de las mujeres no fueron mencionados entre las primeras cuatro por ningún agente 
gubernamental, pero sí por dos encuestados de las ONG. ${ }^{1}$ De 15 funcionarios que dijeron que era su responsabilidad proteger y garantizar los derechos de las comunidades que son reconocidas por las reformas, ninguno señaló que garantizar los derechos de las mujeres, jóvenes u otros grupos vulnerables figuraba entre las actividades emprendidas para lograrlo. No se hizo esta pregunta a los representantes de las ONG, pero sí se les consultó sobre qué medidas deberían tomarse. Uno respondió que garantizar la participación de las mujeres, jóvenes y otros grupos vulnerables en la toma de decisiones era parte de esas medidas.

\section{Diferenciación por género a nivel de las comunidades: membresía en el colectivo}

Uno de los temas más importantes en las reformas de tenencia para las comunidades forestales es identificar quién es el sujeto de la reforma. En los países estudiados, esto varía según el tipo de reforma. Por ejemplo, en las reformas de forestería social en Indonesia, el 'jefe del hogar' representa a la familia que participa en la iniciativa. Por el contrario, en una iniciativa de manejo forestal comunitaria de Uganda, los individuos se unen, por lo tanto algunas veces las mujeres han organizado sus propios grupos de usuarios del bosque, y otros son mixtos. Como se define en la Ley de Comunidades Nativas de Perú (No. 22175), toda la comunidad es el sujeto de la reforma. Así, la manera en que se reconocen los derechos de las mujeres como miembros de este colectivo es primordial - en relación a su condición como miembros de la comunidad y a los órganos colectivos de toma de decisiones.

Aunque los informantes clave en las comunidades estudiadas señalan con frecuencia que las normas establecen derechos equitativos entre hombres y mujeres para participar en los procesos de toma de decisiones, tanto con voz y voto en las asambleas comunales, a menudo, los estatutos de la comunidad se refieren a los miembros sin aclarar específicamente los derechos de las mujeres y de los jóvenes en la toma de decisiones. La ONG de mujeres indígenas rurales, ONAMIAP, señala que algunas veces los procesos de formalización han permitido la discusión y esclarecimiento de la membresía en los estatutos comunitarios (Bolaños 2017).

El derecho de las mujeres a la tierra. En algunas comunidades de Perú, la tierra se otorga tanto al esposo como a la esposa y en otras comunidades, tienen parcelas separadas. En una comunidad nativa en la región del río Napo ${ }^{2}$ (Loreto), las mujeres hablaban con orgullo sobre su trabajo junto a sus esposos. De acuerdo a los informantes clave, en la comunidad existe una percepción generalizada de que hombres y mujeres tienen iguales derechos a la tierra, independientemente de su estado marital. En una comunidad de nuestro estudio, en la región del

\footnotetext{
1 En este punto, no queda claro si los funcionarios de gobierno no escuchan esas preocupaciones o si las 'comunidades' - o más concretamente, las personas de esas comunidades con las que los funcionarios se comunican - no las expresan. 2 Se decidió no utilizar los nombres de las comunidades por cuestiones de privacidad.
}

río Piedras (Madre de Dios), los informantes explicaron que los estatutos comunales que se adoptaron durante el proceso de formalización permitían que las mujeres solteras tengan acceso a la tierra; antes, ellas debían tener una familia.

Estas normas locales afectan los derechos de las mujeres para manejar la tierra familiar luego de la muerte del esposo o de la separación. Cuando se mencionó, los entrevistados manifestaron que las reglas de herencia permitían que las viudas y los hijos varones y mujeres fueran igualmente elegibles para heredar la tierra, pues esta es, en cualquier caso, comunitaria. No obstante, esto resulta engañoso porque existe tanto tierra comunal como tierra asignada a las familias. En la mayoría de comunidades consultadas, los informantes sugirieron que las decisiones sobre derechos de herencia en relación a la distribución de la tierra entre hijos e hijas deberían ser tomadas a nivel del hogar.

En la práctica, algunas de estas reglas varían según las presiones externas sobre la tierra. Por ejemplo, las poblaciones más pequeñas en zonas grandes y remotas (como es común en las comunidades de la región del río Napo) son proclives a tener menos restricciones que las comunidades con poblaciones grandes y cercanas a las carreteras (como es usual en las comunidades nativas en la región del río Bajo Madre de Dios). Estas diferencias son especialmente importantes con respecto al matrimonio.

Matrimonio. Se encontraron diferencias significativas en las comunidades en relación al matrimonio con personas externas a la comunidad. Según los informantes clave, en la mayoría de casos, los nuevos miembros de la comunidad deben pasar por un período de prueba que va desde 6 meses (comunidad nativa en la región de Pebas $^{3}$ ) a 8 años (comunidad nativa en la región del río Alto Madre de Dios). Una de las comunidades permite que personas de fuera de la comunidad se incorporen sin pasar por un período de prueba, el único requisito es trabajar en la comunidad (comunidad nativa en la región del río Piedras). En algunos casos, las mujeres se pueden volver miembros de la comunidad más rápidamente que los hombres, luego de casarse y sin pasar por un período de prueba, aunque su participación en la toma de decisiones puede estar sujeta a la aprobación de la asamblea comunal.

Sin embargo, en dos comunidades (una en la región del río Bajo Madre de Dios y otra en la región del río Alto Madre de Dios), si una mujer contrae matrimonio con un hombre foráneo, tiene que renunciar a todos sus derechos (Quaedvlieg 2017). Estas dos comunidades están sujetas a mayor presión externa sobre la tierra en comparación con las otras que fueron parte del estudio. La justificación para esta medida extrema, que se aplica solo en caso de matrimonio con personas externas que no son indígenas, es evitar que los colonos tomen el control de la tierra comunal, como se ha visto en otros lugares. No obstante, esta regla no se aplica a los hombres que se casan con una mujer de fuera de la comunidad, y las mujeres consideran que este trato diferenciado es injusto.

3 Según el reporte de campo, las personas externas a la comunidad que se encuentran en período de prueba deben participar, sin derecho a tomar decisiones, en todas las mingas (trabajo comunitario), asambleas y reuniones. 


\section{Género y bosques}

Mujeres y hombres usan los bosques de formas diferentes (ver Figura 1). En Loreto, las mujeres participan más en la extracción de productos forestales que en Madre de Dios. Los datos de las entrevistas realizadas en los grupos focales sugieren que las mujeres extraen productos forestales no maderables como aguaje, chonta, tamishi, ungurahui y leche huayo, mientras que los hombres también participan en estas actividades extractivas, pero tienden a involucrarse más en la caza y la pesca.

En Madre de Dios, tanto hombres como mujeres participan en la producción de castaña ${ }^{4}$ (cosecha, secado y pelado) y en algunas comunidades, las mujeres están involucradas de manera intensiva en la producción de artesanías, a menudo con el apoyo de una ONG (la que varía según región y comunidad).

Por supuesto, el acceso al mercado es mayor en algunas comunidades, como las del río Bajo Madre de Dios, que en otras. Los informantes clave resaltaron que las mujeres están desempeñando un rol mayor en las actividades de manejo forestal que en el pasado. Los resultados de la encuesta de hogares muestran que más hombres (60\%) que mujeres (40\%) en comunidades tituladas perciben que las condiciones del bosque han empeorado, lo que podría explicarse debido a que los hombres relacionan las condiciones forestales con la disponibilidad de especies maderables de valor comercial.

Los resultados de la encuesta también encontraron diferencias entre hombres y mujeres en relación a las reglas y normas forestales (ver Figura 2), que tienden a formalizarse después de la titulación, ya que la elaboración y registro de los estatutos de la comunidad son parte de los requerimientos legales. Aunque hay algunas diferencias entre las dos regiones, las más importantes se producen entre hombres y mujeres de las comunidades tituladas. Cuando se desagrega por género y estado de la reforma, la información indica que los

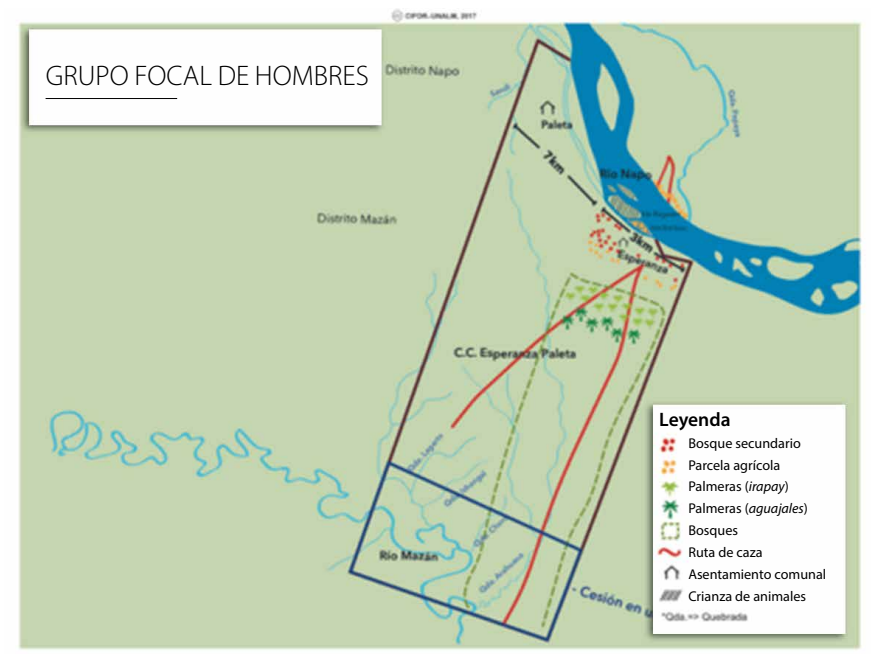

hombres en las comunidades tituladas ${ }^{85}$ perciben las reglas para el uso de recursos y acceso forestal como claras y fáciles de entender (66\%) en comparación con solo 43\% de las mujeres encuestadas. En cuanto a si estas reglas son justas, 58\% de los hombres encuestados en las comunidades tituladas piensan que sí, en comparación con solo 36\% de las mujeres. En general, se evidencian mayores diferencias de género en Madre de Dios, donde todas las comunidades estudiadas estaban tituladas y una porción mucho más grande de hombres percibía que las reglas eran bien conocidas (62\% de hombres versus 38\% de mujeres), claras (74\% de hombres en comparación con 30\% de mujeres) y justas (64\% de hombres y 18\% de mujeres). Esta diferencia no era evidente en Loreto, donde el género parece ser un factor menos importante en la influencia de las percepciones.

En todas las comunidades estudiadas, los hombres participan más que las mujeres en las actividades de manejo forestal comunal, con diferencias significativas en las proporciones que participan en la elaboración de normas (43\% de hombres en comparación con $22 \%$ de mujeres) y en vigilancia forestal (28\% de hombres y $14 \%$ de mujeres) (Cruz-Burga et al. 2018). En las comunidades tituladas, la participación es mayor en ambas actividades, pero las diferencias permanecen: $55 \%$ de los hombres señala tomar parte en la elaboración de normas versus solo 37\% de las mujeres. En las comunidades sin título, el nivel de participación es bajo tanto para hombres (14\%) como para mujeres (15\%).

De modo interesante, las mujeres dicen participar más que los hombres en la supervisión del cumplimiento de las normas en las comunidades tituladas (30\% de mujeres y $24 \%$ de hombres), y la diferencia es aún mayor en las comunidades no tituladas (41\% de mujeres, 13\% de hombres). En relación al arbitraje de disputas, la participación de las mujeres es mayor que la de los hombres, tanto en las comunidades con título (40\% de mujeres, $29 \%$ de hombres) y sin titular (40\% de mujeres, $21 \%$ de hombres). Las razones para estas diferencias requieren mayor investigación.

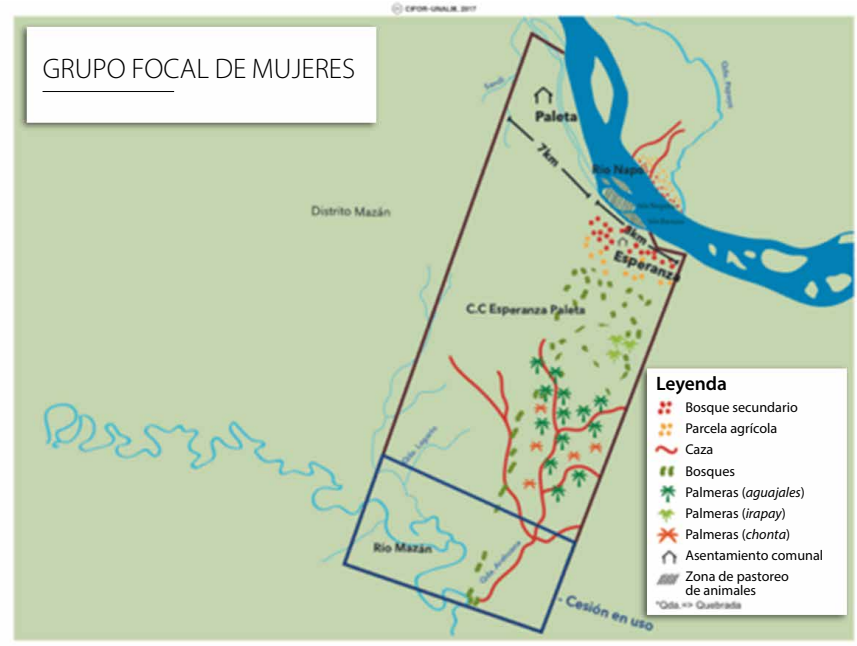

Figura 1. Mapas participativos que analizan los usos de la tierra (producidos durante la discusión de grupo focal en una comunidad de la región del río Napo, realizado por CIFOR y la Universidad Nacional Agraria La Molina en 2015)

4 Cerca de la mitad de las comunidades estudiadas en Madre de Dios tienen árboles de castaña.
5 La información corresponde a 835 personas consultadas en total (hombres y mujeres) de comunidades tituladas tanto en las regiones de Loreto y Madre de Dios. 


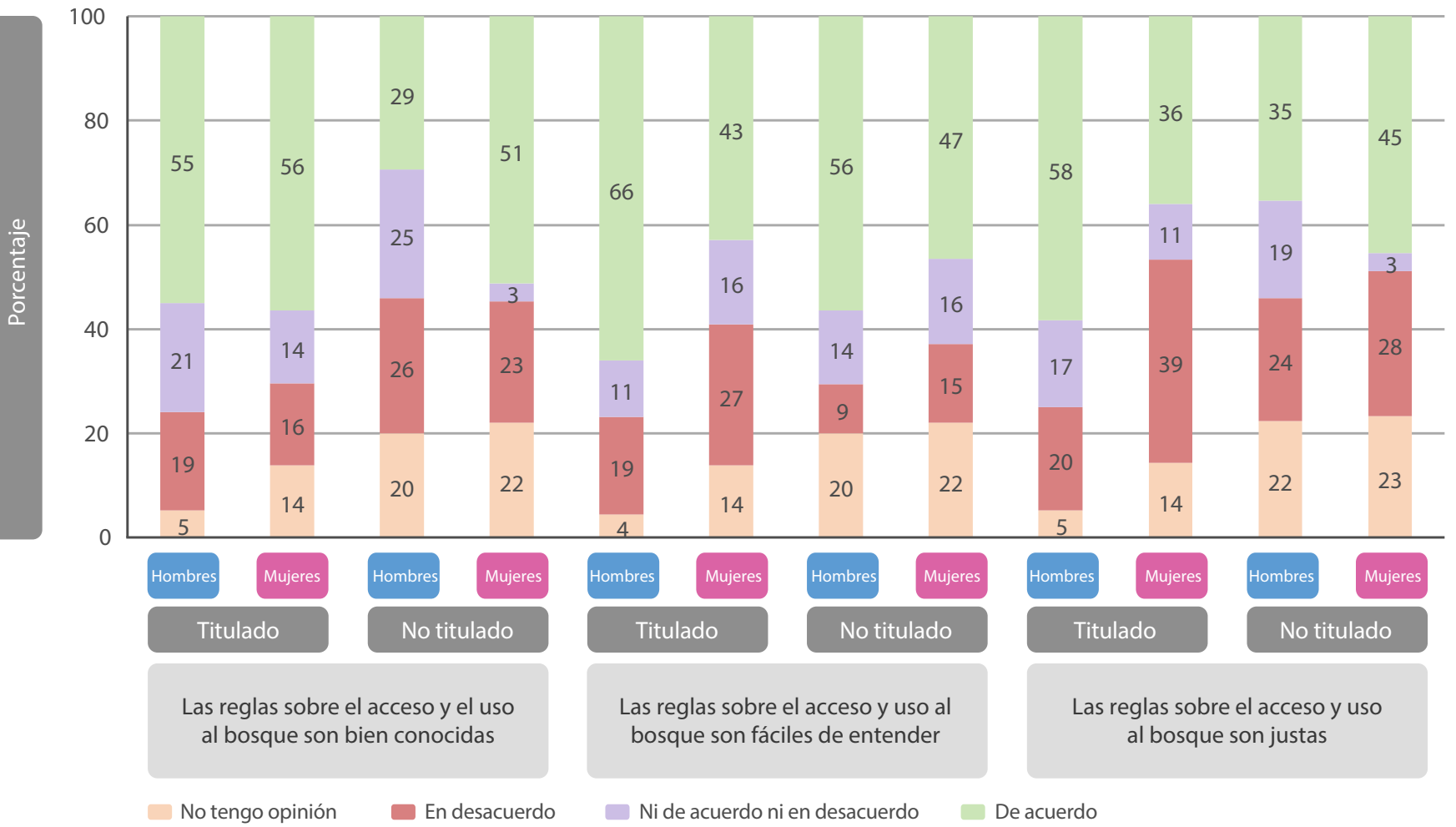

Figura 2. Percepción de las reglas sobre el acceso y uso de recursos forestales en comunidades tituladas y no tituladas ( $n=1006)$

\section{Las mujeres en la toma de decisiones}

En los grupos focales y entrevistas, tanto hombres como mujeres afirmaron que la participación de las mujeres en la toma de decisiones se ha incrementado marcadamente en las dos últimas décadas. Una comunidad en la región de Napo, en Loreto, mencionó que actualmente tiene una jefa comunal y que anteriormente también tuvo otra, ambas en los últimos 10 años, sin embargo, se trata de un caso todavía bastante excepcional. Este avance se atribuye en parte a los procesos de formalización de la comunidad y también a un declive de la cultura machista, lo que permite un mayor respeto por las mujeres y puede estar relacionado a organizaciones eclesiásticas y a las ONG en la región, que están introduciendo nuevas perspectivas sobre participación y equidad. Sin embargo, la participación de las mujeres en los puestos de liderazgo usualmente se basa en estereotipos ligados a actividades reproductivas domésticas, tradicionalmente asociadas con ellas (Durán et al. 2018).

Los resultados de la encuesta de hogares muestran que en las comunidades tituladas, 93\% de los hombres han participado en reuniones vinculadas al uso y manejo forestal, mientras que un $77 \%$ de las mujeres dijo haber participado al menos una vez. Estos porcentajes son menores en las comunidades no tituladas: $82 \%$ de los hombres y $51 \%$ de las mujeres tomaron parte. A nivel regional, existe una mayor participación en Madre de Dios (95\% de los participantes reportó algún nivel de involucramiento) que en Loreto (75\%).

\section{Seguridad e inseguridad de tenencia}

En talleres de análisis prospectiva participativa que analizaban los escenarios futuros para la seguridad de la tenencia con mujeres indígenas de todo el país, se identificaron un número de factores clave o fuerzas impulsoras que influencian la seguridad de tenencia. Algunas de ellas eran similares a aquellas que se obtuvieron en talleres mixtos y que eran dominados por los hombres, tales como falta de financiamiento, de capacidades y habilidades de empoderamiento en las organizaciones comunales, requerimientos legales para acceder a los recursos naturales en las áreas tituladas, carencia de voluntad política con relación a cuestiones indígenas, así como prioridades de desarrollo que no coinciden con las perspectivas de los pueblos indígenas (Zamora y Monterroso 2017).

Otras prioridades eran diferentes y fueron mencionadas solo por las mujeres, tales como: (1) la necesidad de incluir equidad de género en la implementación de políticas nacionales y regionales, por medio de mecanismos que recojan las percepciones sobre su territorio tanto de hombres como de mujeres. (2) El fuerte impacto de la violencia que prevalece en las comunidades, particularmente de las actividades extractivas en los territorios indígenas. (3) La necesidad de afianzar la identidad y la moral indígena dentro de las familias y la comunidad como aspecto central al fortalecimiento del manejo y gobernanza de sus territorios. (4) La necesidad de reforzar los mecanismos de adaptación al cambio climático. El debate sobre el empoderamiento de las mujeres y los espacios de 
participación sacaron a la luz la necesidad de asignar fondos para actividades que aborden específicamente temas de interés de las mujeres, como el desarrollo de capacidades y empoderamiento.

Adicionalmente, las mujeres argumentaron la necesidad de incluir discusiones y actividades de sensibilización sobre género a nivel de la comunidad, para integrar las perspectivas de las mujeres indígenas en las agendas regionales, incluyendo el cumplimiento de las cuotas de género en la participación política.

Estas diferencias en las prioridades podrían explicar algunos de los resultados sobre la seguridad de tenencia en la encuesta de hogares, a pesar de que, en términos generales, la mayoría de hombres y mujeres en las comunidades con titulación se sentían razonablemente seguros de sus derechos en dos de tres preguntas (ver Figura 3).

En todos los países del estudio, Perú reporta la mayor proporción de encuestados (80\%) que está de acuerdo con el enunciado: "Desde que mi comunidad fue titulada, siento que mis derechos y acceso a la tierra y a los bosques son sólidos y seguros". En las comunidades tituladas, la proporción de hombres que percibe sus derechos como sólidos y seguros (86\%) es mayor que la de las mujeres (76\%). Las pruebas estadísticas (chi-cuadrado de Pearson) señalan que esta diferencia es significativa $(p<0.01)$. También existen diferencias regionales importantes: $82 \%$ de los encuestados en Madre de Dios está de acuerdo con la afirmación, mientras que solo el 55\% de los consultados en Loreto está de acuerdo.
Los resultados del análisis sobre si los encuestados consideran que "las condiciones de seguridad de la tenencia han mejorado durante los últimos 20 años" también son acordes. En las comunidades tituladas, mientras 56\% de los hombres encuestados señalan que las condiciones de seguridad de tenencia han mejorado, solo 39\% de las mujeres están de acuerdo. La diferencia es estadísticamente significativa $(p<0.01)$. A pesar de que en las comunidades tituladas hay una percepción bastante positiva de que "los derechos se protegerán y se harán cumplir" (para el 81\% de los hombres y el 75\% de las mujeres), cuando se preguntó sobre la "preocupación de que alguien pueda disputar sus derechos de acceso, uso y manejo de la tierra y recursos de la comunidad", 43\% de los hombres encuestados indicaron que no se sienten preocupados, en comparación con solo el 22\% de mujeres. Esta diferencia de género también es significativa estadísticamente $(p<0.05)$.

Estos resultados concuerdan con la percepción sobre si la formalización de derechos por medio de la titulación ha producido beneficios como, por ejemplo, si las condiciones de subsistencia han mejorado (ver Figura 3). En este caso, solo $57 \%$ de los hombres y $49 \%$ de las mujeres participantes están de acuerdo con que la titulación ha conducido a obtener mayores ingresos o mejoras en los medios de vida. Estos resultados resaltan que aunque la titulación es una fuente importante para la seguridad de tenencia, no brinda automáticamente mejoras en los medios de vida, y que factores como este pueden también influenciar la percepción de seguridad, incluyendo las diferencias de género.

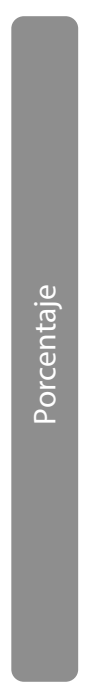

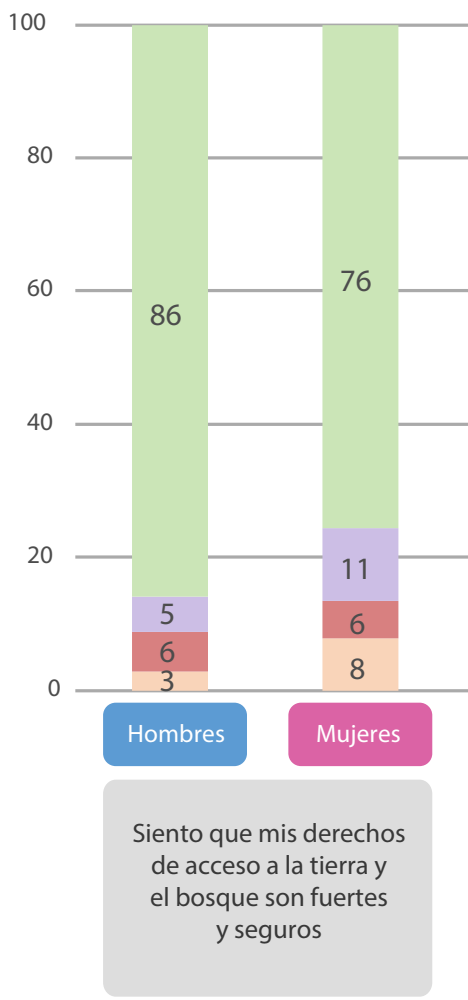

No tengo opinión

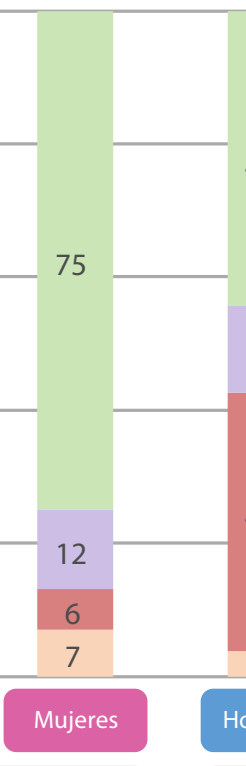

Si tengo una disputa relacionada con mis derechos de uso y acceso al bosque, tengo confianza de que mis derechos estarán protegidos

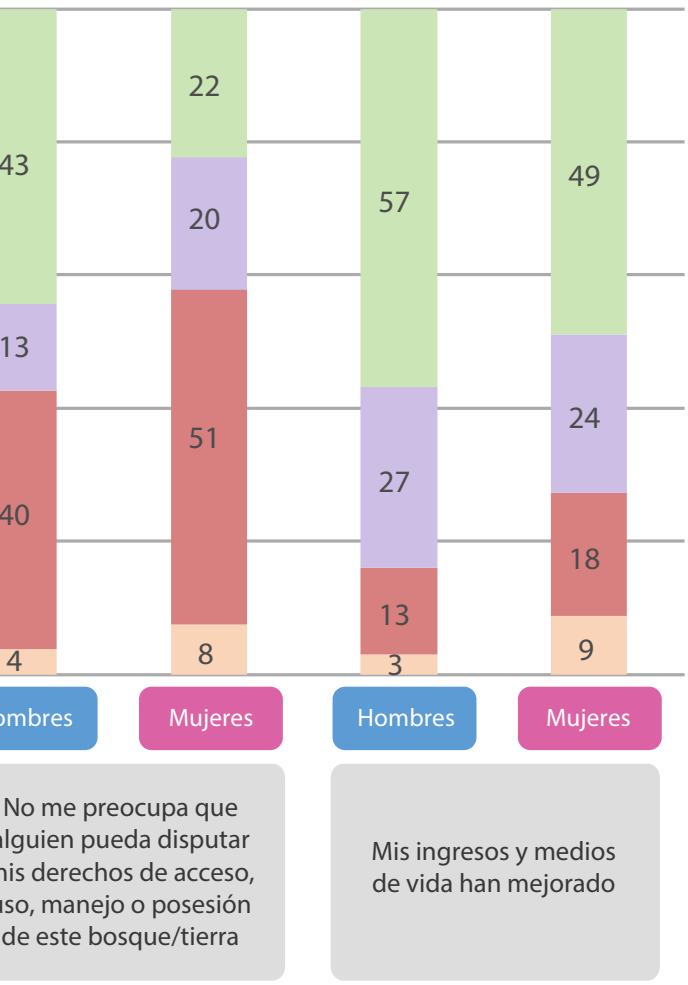

alguien pueda disputar mis derechos de acceso, uso, manejo o posesión de este bosque/tierra
Mis ingresos y medios de vida han mejorado 


\section{Discusión y conclusiones}

Los resultados presentados aquí plantean preguntas importantes sobre la tenencia de tierras y bosques para las mujeres, tanto en términos del rol del Estado y de la comunidad, así como la relación entre ambos.

Los resultados demuestran que pocas mujeres participan en el proceso administrativo para formalizar los derechos de las comunidades nativas a la tierra. Unas pocas personas en el aparato gubernamental ven específicamente los derechos de las mujeres como una meta o asumen la exclusión de las mujeres como un problema que necesita abordarse. En realidad, y en forma general, la sensibilización sobre género o, específicamente, la percepción de que las mujeres pueden tener necesidades y expectativas específicas de las reformas o requieren atención en particular- es muy baja entre los funcionarios gubernamentales entrevistados. Las respuestas, de las ONG en Perú y de funcionarios públicos en otros países, en alguna medida, brindan evidencia comparativa para este análisis. Tener más mujeres en estas posiciones técnicas de gobierno (a pesar de que estos otros países tienen incluso menos que Perú) puede ser de ayuda, pero es tan importante como generar más sensibilización sobre el tema de género y a la vez considerar otros recursos de diferenciación social, como la edad.

Debido a que las leyes nacionales protegen la equidad y la inclusión e incorporan iguales derechos a la tierra para mujeres y niñas, la existencia de garantías para asegurar la membresía a la comunidad tendría sentido no solo en las comunidades campesinas, sino también en las comunidades nativas. A fin de cuentas, si según las leyes el Estado tiene una obligación con las mujeres en las comunidades campesinas, ¿por qué no se hace también en las comunidades nativas?

La predisposición a ver a las comunidades nativas solo como un colectivo lleva a perder una oportunidad en los procesos de formalización, y a la incapacidad de garantizar la igualdad de derechos de las mujeres en virtud de la ley. Esto permite que los derechos de las mujeres sean definidos solo por sus comunidades. El caso de las comunidades nativas en la región de Madre de Dios- donde por primera vez se adoptaron nuevos estatutos para permitir que las mujeres solteras tengan acceso a la tierra- es un claro ejemplo de lo que la formalización podría hacer por las mujeres. Los derechos de membresía claros para las mujeres dentro de estos colectivos tienen implicancias importantes en las formas en que las mujeres pueden participar en los espacios de toma de decisiones para reforzar más aún sus derechos de acceso y uso de los recursos de la comunidad.

En función de las entrevistas realizadas en la comunidad, los derechos equitativos a la tierra y a la herencia parecen ser la norma, al menos en teoría. Sin embargo, en el lenguaje utilizado por los informantes se entendía que se trataba de una opción, no de una norma. Ocasionalmente, las reglas de matrimonio benefician más a las mujeres que a los hombres, pero a veces pueden ser extremas para ellas e incluir la pérdida de todos sus derechos. La preocupación sobre la pérdida de los derechos comunales, así como de las costumbres y la cultura a causa de la infiltración de personas externas no es infundada (ver, por ejemplo, Grann 2017). Sin embargo, existen soluciones que podrían limitar el poder de externos sin privar de derechos a las mujeres de la comunidad.

Diferencias importantes en el uso forestal (con áreas de uso destacadas en los mapas así como la percepción y justicia de las reglas y normas forestales) destacan la importancia del análisis de género.

A veces, las diferencias de género son ignoradas o se asume que un jefe de hogar puede hablar y hablará por los intereses de todos. La historia sugiere que este no es siempre el caso y debe alertar a todos los que trabajan en temas como derechos de tenencia de la tierra y los recursos, demarcación de tierras así como en establecimiento y cumplimiento de reglas. No entender claramente estas diferencias puede llevar a la pérdida de derechos de las mujeres y, potencialmente, de otros grupos vulnerables, así como a la inseguridad alimentaria de sus familias.

Con base en el análisis de los resultados a nivel de comunidad, hay pocas mujeres en posiciones de liderazgo en la comunidad. Esto apunta a la necesidad de aumentar la participación de las mujeres en la toma de decisiones y en la gobernanza comunitaria. En la práctica, aún existe resistencia considerable y un largo camino por delante en materia de equidad de género. Cuando las mujeres se encuentran en reuniones, separadas de los hombres, como en el taller de escenarios sobre la seguridad e inseguridad de tenencia, a menudo expresan inquietudes que no se han planteado en los foros mixtos. Al mismo tiempo, cabe señalar que frecuentemente las mujeres son cuidadosas para aclarar que la cuestión no es poner a las mujeres por encima de los hombres o respaldar procesos de formalización individuales, sino resaltar las perspectivas tanto de los hombres como de las mujeres en la discusión de la gobernanza de las tierras de las comunidades nativas.

Las mujeres son afectadas por la seguridad de tenencia de la tierra colectiva pero también por su estatus específico como mujeres (que además les afecta de forma diferente, según otros factores como la condición social). De acuerdo a algunos indicadores, la gran mayoría de mujeres en nuestro estudio siente que sus derechos de tenencia son seguros, aunque un porcentaje significativamente menos que los hombres. Los resultados subrayan la necesidad de desagregar las percepciones por género. Además, sugieren la necesidad de realizar un mayor análisis para entender más ampliamente cómo las mujeres y hombres perciben las amenazas externas, así como su participación en la toma de decisiones, especialmente en la elaboración de normas. Esto nos lleva de regreso a la necesidad de revisar las reglas sobre membresía, que al final actúan como barrera para la participación de las mujeres. También plantea la pregunta sobre quién lleva la 
carga de las tareas de vigilancia y cumplimiento de las normas, independientemente de si las mujeres participan o no en la elaboración de éstas.

Algunas formas para avanzar en el tema incluyen el desarrollo de capacidades para mujeres, así como capacitación y reflexión en materia de género para funcionarios gubernamentales, federaciones y comunidades indígenas. Una mayor articulación entre los funcionarios de gobierno y comunidades, con el respaldo de las ONG, organizaciones de mujeres y federaciones, será esencial para mejorar las políticas de formalización de manera que aseguren la participación de las mujeres en estos procesos y protejan los derechos a la tierra y a los recursos en beneficio de las familias y comunidades indígenas.

\section{Agradecimientos}

El Estudio Comparativo Global sobre Reformas de Tenencia en Tierras Forestales de CIFOR es financiado por la Comisión Europea y el Fondo para el Medio Ambiente Mundial (FMAM) y cuenta con el respaldo técnico del Fondo Internacional para el Desarrollo Agrícola (FIDA) y la Organización de las Naciones Unidas para la Alimentación y la Agricultura (FAO). También agradecemos a Safia Aggarwal, Omaira Bolaños y Rosaluz Durán por sus útiles revisiones y comentarios. Este estudio forma parte del Programa sobre Políticas, Instituciones y Mercados (PIM), dirigido por el Instituto Internacional de Investigación sobre Políticas Alimentarias (IFPRI); y el Programa de Investigación del CGIAR sobre Bosques, Árboles y Agroforestería (FTA), liderado por CIFOR. Las opiniones aquí expresadas pertenecen a las autoras y no reflejan necesariamente los puntos de vista de CIFOR, CGIAR o los patrocinadores financieros.

\section{Referencias}

Bolaños O. 2017. Resumen político: Los derechos de las mujeres indígenas y los desafíos para los proyectos de titulación de la propiedad comunal en el Perú. Lima: RRI, ONAMIAP, CIFOR.

Bourgeois R, Liswanti N, Zamora A, Mukasa C, Herawati T, Monterroso I, Mshale B, Banjade MB, Mwangi E y Larson A. 2017. Guide for co-elaboration of scenarios: Facilitating future oriented shared understanding and joint action in the context of forest tenure reforms and tenure security. Bogor, Indonesia: CIFOR.

Cruz-Burga Z, Monterroso I, Larson AM, Valencia F y Saldaña J. 2018. El impacto de la formalización de los derechos sobre la tierra y el bosque. Perspectiva de comunidades en Madre de Dios y Loreto. Infobrief. Lima: CIFOR.

Durán R, Monterroso I y Larson AM. 2018. Género e interculturalidad en la formalización de las comunidades nativas en el Perú. Lima: CIFOR.

Grann D. 2017. Killers of the Flower Moon. New York: Knopf Doubleday.

Keene S y Ginsburg C. 2017. Power and potential. A comparative analysis of national laws and regulations concerning women's rights to community forests. Washington: Resources and Rights Initiative.

Monterroso I, Larson AM, Quaedvlieg J, Valencia F, Jarama L y Saldaña J. 2018. Formalización del derecho colectivo de las comunidades nativas en Perú: La perspectiva de los funcionarios que la implementan. Infobrief. Bogor, Indonesia: CIFOR.

Monterroso I, Cronkleton P, Pinedo D y Larson AM. 2017. Reclaiming collective rights: Land and forest tenure reforms in Peru (1960-2016). Working Paper 224 Bogor, Indonesia: CIFOR.

Monterroso I y Larson AM. 2018. Avances del proceso de formalización de derechos de comunidades nativas en la Amazonía peruana (2014-2018). Infobrief No. 233. Bogor, Indonesia: CIFOR.

Notess L, Veit PG, Monterroso I, Sulle AE, Larson AM, Gindroz AS, Quaedvlieg J y Williams A. 2018. The scramble for land rights: Reducing inequity between communities and companies. Washington: WRI, CIFOR, AsM Law Office, UCRT, RFUS.

Quaedvlieg J. 2017. Qualitative data analysis in Uganda, Indonesia and Peru. A Global comparative study of design and implementation of forest tenure reform. Consultancy report. Bogor, Indonesia: CIFOR.

[SICNA-IBC] Sistema de Información sobre Comunidades Nativas de la Amazonía Peruana - Instituto del Bien Común. 2016. Sistema de información sobre comunidades nativas de la Amazonía Peruana. Perú: IBC. Consultado el 1de setiembre de 2018. http://www.ibcperu.org/servicios/sicna/

Soria O. 2017. Recommendations for monitoring improvement of Peruvian communal land tenure legislation. Consultancy report. Lima, Perú: CIFOR.

Zamora A y Monterroso I. 2017. Visiones regionales y locales sobre la inseguridad de tenencia en Loreto y Madre de Dios. Infobrief No. 198. Bogor, Indonesia: CIFOR

\section{\begin{tabular}{|l|l|l|l}
\hline RESEARCH & El programa sobre Políticas, Instituciones y Mercados (PIM) del CGIAR, lidera investigación orientada a la acción \\
PROGRAM on & para informar a los tomadores de decisión con la evidencia necesaria para desarrollar políticas alimentarias y \\
Policies, & agrícolas que puedan servir de mejor manera los intereses de los productores y consumidores, tanto hombres y \\
Institutions, & mujeres. PIM combina los recursos de los centros de investigación del CGIAR, así como numerosos socios a nivel \\
internacional, regional y nacional. El programa es liderado por el el Instituto Internacional de Investigación sobre & Políticas Alimentarias (IFPRI, por sus siglas en inglés). www.pim.cgiar.org
\end{tabular}}

\begin{tabular}{l|ll} 
PROGRAMA DE & El Programa de Investigación de CGIAR sobre Bosques, Árboles y Agroforestería (FTA) es el programa de \\
INVESTIGACIÓN SOBRE & $\begin{array}{l}\text { investigación para el desarrollo más grande del mundo, dedicado a mejorar el papel de bosques, árboles y la } \\
\text { Bosques, Árboles y y }\end{array}$ \\
agroforestería para el desarrollo sostenible, la seguridad alimentaria, y frente al cambio climático. CIFOR dirige el \\
programa FTA en asociación con Bioversity International, CATIE, CIRAD, ICRAF, INBAR y TBI. \\
La investigación del Programa FTA cuenta con el apoyo del Fondo Fiduciario del CGIAR: cgiar.org/funders
\end{tabular}

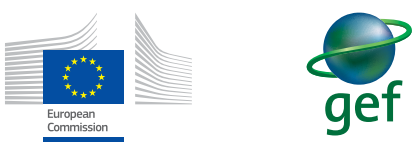

cifor.org

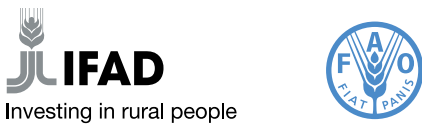

Organización de las Naciones Unidas para la Alimentación y la Agricultura 